\title{
Distribution and dispersal of anguillid leptocephali in the western Pacific Ocean revealed by molecular analysis
}

\author{
Jun Aoyama ${ }^{1, *}$, Noritaka Mochioka ${ }^{2}$, Tsuguo Otake ${ }^{1, * *}$, Satoshi Ishikawa ${ }^{1}$, \\ Yutaka Kawakami $^{2, * * *}$, Peter Castle ${ }^{3}$, Mutsumi Nishida ${ }^{4, * * *}$, Katsumi Tsukamoto ${ }^{1}$ \\ 'Ocean Research Institute, University of Tokyo, 1-15-1 Minamidai, Nakano, Tokyo 164-8639, Japan \\ ${ }^{2}$ Department of Fisheries, Faculty of Agriculture, Kyushu University, 6-10-1, Hakozaki, Higashi-ku, Fukuoka 812, Japan \\ ${ }^{3}$ School of Biological Sciences, Victoria University of Wellington, PO Box 600, Wellington 6000, New Zealand \\ ${ }^{4}$ Department of Marine Bioscience, Fukui Prefectural University, 1-1 Gakuen-cho, Obama, Fukui 917-0003, Japan
}

\begin{abstract}
To determine the distribution and dispersal of leptocephalus larvae of freshwater eels Anguilla spp. in the western Pacific, mitochondrial DNA sequences of 63 larvae were compared with those of adults. All larvae were definitively identified as 1 of the following 6 species: $A$. marmorata, $A$. bicolor pacifica, A. reinhardti, A australis, A. megastoma or A. japonica. Species-specific patchiness in the distribution of these leptocephali in the western Pacific was apparent. A. marmorata showed 2 different areas of patchiness in both the North and South Pacific (around $15^{\circ} \mathrm{N}$ and $10^{\circ} \mathrm{S}$ ). Horizontal distributions of anguillid leptocephali are apparently closely related to the freshwater habitat of adults by way of ocean currents. Accordingly, specific dispersal routes of leptocephali from likely spawning areas to their freshwater habitats are suggested.
\end{abstract}

KEY WORDS: Leptocephali - Anguilla - Western Pacific - DNA identification - Dispersal - Spawning areas

\section{INTRODUCTION}

Based on extensive earlier research it has become well known that freshwater eels Anguilla spp. are catadromous and have a long period of larval oceanic dispersal. The collection of great numbers of anguillid leptocephali in the open ocean suggests that the North Atlantic species $A$. anguilla and $A$. rostrata spawn in the Sargasso Sea (Schmidt 1923, Kleckner \& McCleave 1985), and the Japanese eel A. japonica to the west of the Mariana Islands (Tsukamoto 1992). However, in the South Pacific, where 6 species occur (Ege 1939,

•E-mail: jaoyama@ori.u-tokyo.ac.jp

Present addresses:

- Faculty of Bioresources, Mie University Kamihama-cho, Tsu, Mie 514-8507, Japan

-..Faculty of Fisheries, Hokkaido University 3-1-1 Minatocho, Hakobate, Hokkaido 041-8611, Japan

-..Ocean Research Institute, University of Tokyo, 1-15-1, Minamidai, Nakano, Tokyo 164-8639, Japan
Jespersen 1942), the spawning areas and larval dispersal routes of these species are still unknown. Only 16 anguillid leptocephali have been reported so far from this part of the Pacific: $2 \mathrm{~A}$. megastoma, 4 A. australis, 3 A. obscura and 7 A. marmorata / A. reinhardtii (Jespersen 1942, Castle 1963).

The leptocephalus larva, which is the unique larval form of elopomorph fishes, is so distinct from the adults that their study almost constitutes a separate science (Smith 1989). Even for leptocephali of Anguilla, which have been well described from the morphological characteristics of their adults, identification to species level is often difficult, especially for small specimens because their morphological keys are not fully developed (Castle 1963, Mochioka 1996).

Molecular techniques, which have recently seen great advancements, are able to identify characters which continue on from the larva through the metamorphosis to the adult. These molecular characters, determined from fully developed adults, are likely to 
be a powerful tool for the identification of small leptocephali which are not well developed morphologically.

In this paper we identified western Pacific anguillid leptocephali from mitochondrial DNA sequences and revealed their horizontal distributions and oceanic movements. Based on these results it might now be possible to explore further the spawning area(s) for the Australian and New Zealand short-finned eel Anguilla australis in particular.

\section{MATERIALS AND METHODS}

Materials. A total of 372 anguillid leptocephali were collected during the research cruise KH95-2 of RV 'Hakuho-Maru' of the Ocean Research Institute, University of Tokyo in the western Pacific (Fig. 1). The leptocephali were sampled by Isaacs-Kidd Midwater Trawl (IKMT) with a $8.7 \mathrm{~m}^{2}$ mouth opening and $0.5 \mathrm{~mm}$ mesh. Net sampling involved a 60 min oblique tow from the surface to $300 \mathrm{~m}$ depth. The following morphological characters of fresh specimen were recorded using a binocular microscope: total length (to the nearest $0.1 \mathrm{~mm}$ ), total number of myomeres, positions of the origin of dorsal and anal fins, anterior margin of gall bladder, and vertical blood vessels. The identification of species was made based on these morphological characters, following Jespersen (1942), Castle (1963) and Tabeta \& Mochioka (1988). A total of 128 leptocephali were definitively identified as Anguilla japonica by morphological characters, and separated from the total of 372 . Of the remaining 244,63 specimens, comprising all morphological types, were subsampled and preserved in $95 \%$ ethanol or in a deep freezer at $-80^{\circ} \mathrm{C}$ for subsequent genetic analysis (Table 1 ). The specimens which could not be examined genetically were considered as unidentified specimens.

DNA analysis. Total genomic DNA extraction from each leptocephalus was carried out following a standard protocol (Aoyama \& Tsukamoto 1997). A portion of mitochondrial $16 \mathrm{~S}$ ribosomal RNA gene was amplified via polymerase chain reaction (PCR) using the oligonucleotide primers $\mathrm{H} 2510$ and H3058 (Miya \& Nishida 1996). Amplification parameters were 35 cycles of denaturation at $94^{\circ} \mathrm{C}$ for $30 \mathrm{~s}$, annealing at $55^{\circ} \mathrm{C}$ for $30 \mathrm{~s}$ and extension at $72^{\circ} \mathrm{C}$ for $60 \mathrm{~s}$. Double-stranded DNA products from PCR were sequenced following the manufacture's protocol (Applied Biosystems, Inc.) on a 373A DNA sequencer (Applied Biosystems, Inc.). Sequences were obtained from light strand only.

Species identification by mitochondrial DNA sequences. Using MacClade version 3.0 (Maddison \& Maddison 1992), we first compared sequences previously determined from identified adult specimens of the Pacific species Anguilla japonica, A. marmorata,

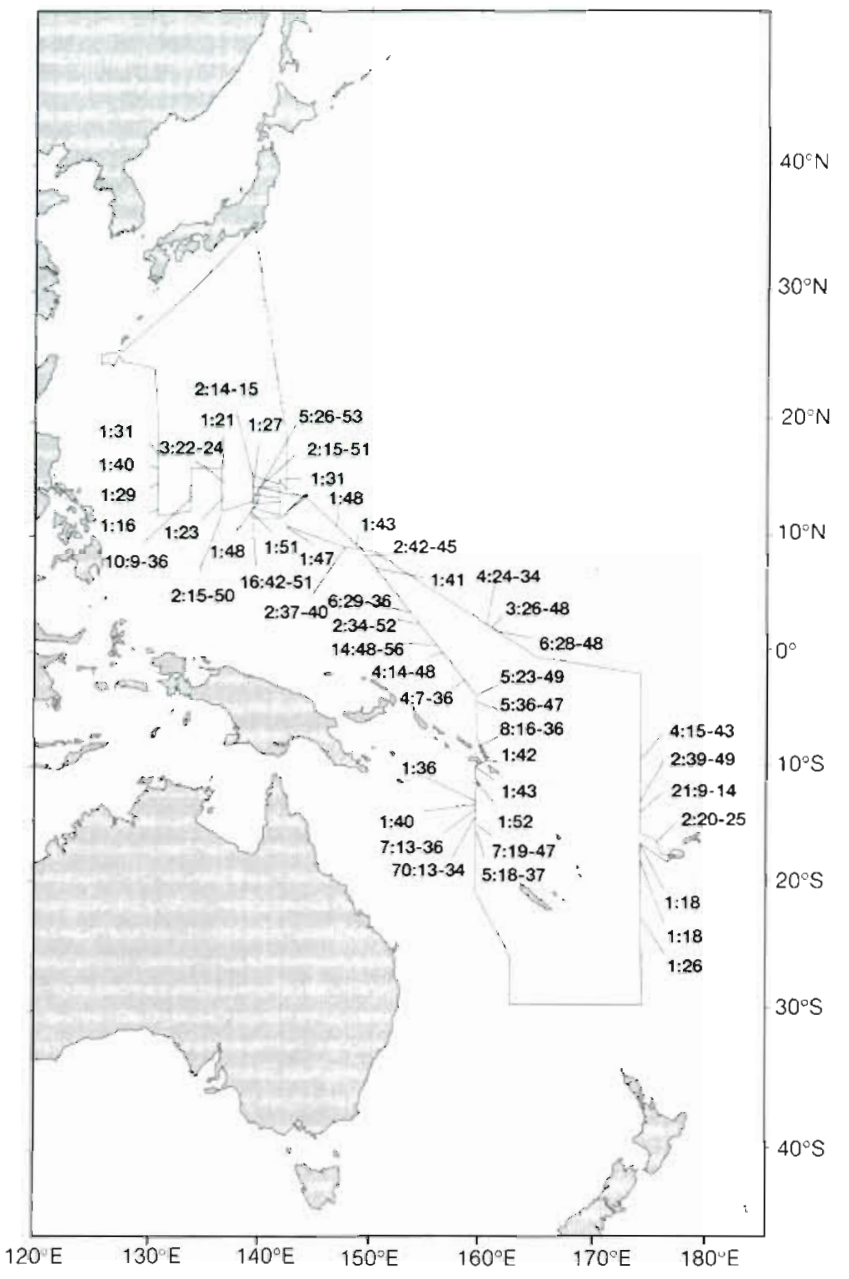

Fig. 1. Chart and sampling locations of anguillid leptocephali, except for 128 Anguilla japonica, collected on the research cruise during 7 July to 27 September 1995 of RV 'HakuhoMaru', Ocean Research Institute, University of Tokyo. Number of leptocephali collected and their ranges of total length $(\mathrm{mm})$ are shown for each sample location

A. borneensis, A. celebesensis, A. interioris, A. megastoma, A. bicolor pacifica, A. reinhardtii, A. obscura, $A$. australis australis, $A$. australis schmidtii and $A$. dieffenbachii (Aoyama 1998). These sequence data will appear in the DDBJ/EMBL/GenBank nucleotide sequence databases with the accession numbers: $\mathrm{AB} 021748, \mathrm{AB}$ $021750-\mathrm{AB} 021754, \mathrm{AB} 021756, \mathrm{AB} 021758$, AB021762$\mathrm{AB} 021762, \mathrm{AB} 021764$.

Based on this comparison, we established 'specific standard sequences' comprised of 60 sites at which specific substitutions were observed (Table 2). Thereafter, homologous sites obtained from leptocephalus sequences were selected for comparison with these standards and the number of differences were counted by PAUP version 3.0 (Swofford 1991). 


\section{RESULTS}

\section{Species identification}

A portion of the mitochondrial $16 \mathrm{~S}$ ribosomal RNA gene (412 to 469 base pairs) was determined from all of the 63 anguillid leptocephali analyzed, and the 60 homologous sites were selected for comparison with specific standard sequences. Each leptocephalus sequence corresponded to one of the specific standard sequences only $(0$ to 3 sites differences), while they were distinguishable from the others with more than 4 -fold differences ( 5 to 28 sites, Table 3). Based on this observation, all of the 63 individuals examined could be precisely identified as follows: 1 Anguilla japonica, 9 A. australis, $25 \mathrm{~A}$. bicolor pacifica, $14 \mathrm{~A}$. marmorata, 2 A. megastoma and 12 A. reinhardtii (Table 1). Other species which might occur around this area are $A$. celebesensis, $A$. borneensis, $A$. obscura and $A$. dieffenbachii, but were not found.

Among 63 leptocephali, 44 were identified morphologically, and of these, $35(79.5 \%)$ agreed with the identification by molecular characters (Table 1).

\section{Distribution of anguillid leptocephali}

Mapping of locations of identified leptocephali revealed that their horizontal distributions showed a specific patchiness (Fig, 2). In the North Pacific, 17 Anguilla bicolor pacifica and 6 A. marmorata were distributed from the equator to $10^{\circ} \mathrm{N}$, and $10^{\circ} \mathrm{N}$ to $20^{\circ} \mathrm{N}$, respectively. In the South Pacific, $2 \mathrm{~A}$. megastoma were found near $10^{\circ} \mathrm{S}$ and $8 \mathrm{~A}$. marmorata from the equator to $10^{\circ} \mathrm{S}$. Nine A. australis and 12 A. reinhardtii were found from $10^{\circ} \mathrm{S}$ to $20^{\circ} \mathrm{S}$. The total length distribution of these specimens did not exhibit significant differences between species (Mann-Whitney $U$-test, p > 0.05, Fig. 3).
Table 1. Results of morphological and genetic identification of anguillid leptocephali collected during the research cruise of RV 'Hakuho-Maru' Ocean Research Institute, University of Tokyo, in 1995. Species names are abbreviated as follows: jap: A. japonica, rei: A. reinhardtii, obs: A. obscura, meg: A. megastoma, mar: $A$. marmorata, die: A. dieffenbachii, bip: $A$. bicolor pacifica, aua: A. australis

\begin{tabular}{|c|c|c|c|c|c|}
\hline $\begin{array}{l}\text { Fish } \\
\text { no. }\end{array}$ & Date & $\underset{(\mathrm{mm})}{\mathrm{TL}}$ & $\begin{array}{l}\text { Morphologically } \\
\text { identified as }\end{array}$ & $\begin{array}{l}\text { Genetically } \\
\text { identified as }\end{array}$ & Sampling location \\
\hline 01 & $18 \mathrm{Jul}$ & 39.6 & mar & mar & $15^{\circ} 59^{\prime} 8 \mathrm{~N}, 130^{\circ} 59^{\prime} 9 \mathrm{E}$ \\
\hline 02 & $18 \mathrm{Jul}$ & 29.4 & mar & mar & $14^{\circ} 60^{\circ} 0 \mathrm{~N}, 131^{\circ} 00^{\prime} 2 \mathrm{E}$ \\
\hline 03 & $19 \mathrm{Jul}$ & 16.3 & mar & mar & $12^{\circ} 60^{\prime} 0 \mathrm{~N}, 131^{\circ} 00^{\prime} 1 \mathrm{E}$ \\
\hline 04 & $21 \mathrm{Jul}$ & 36.0 & mar & mar & $14^{\circ} 00^{\prime} 1 \mathrm{~N}, 133^{\circ} 59^{\prime} 9 \mathrm{E}$ \\
\hline 05 & $23 \mathrm{Jul}$ & 28.1 & jap & jap & $14^{\circ} 00^{\prime} 7 \mathrm{~N}, 136^{\circ} 57^{\prime} 7 \mathrm{E}$ \\
\hline 06 & $23 \mathrm{Jul}$ & 22.0 & mar & $\operatorname{mar}$ & $14^{\circ} 60^{\circ} 0 \mathrm{~N}, 137^{\circ} 00^{\prime} 1 \mathrm{E}$ \\
\hline 07 & $23 \mathrm{Jul}$ & 24.2 & mar & $\operatorname{mar}$ & $14^{\circ} 60^{\circ} 0 \mathrm{~N}, 137^{\circ} 00^{\prime} 2 \mathrm{E}$ \\
\hline 08 & $24 \mathrm{Jul}$ & 49.6 & bip & bip & $13^{\circ} 00^{\prime} 1 \mathrm{~N}, 136^{\circ} 59^{\prime} 9 \mathrm{E}$ \\
\hline 09 & $24 \mathrm{Jul}$ & & bip & bip & $14^{\circ} 03^{\prime} 9 \mathrm{~N}, 139^{\circ} 59^{\prime} 3 \mathrm{E}$ \\
\hline 10 & $25 \mathrm{Jul}$ & 50.5 & bip & bip & $15^{\circ} 00^{\prime} 1 \mathrm{~N}, 140^{\circ} 00^{\prime} 3 \mathrm{E}$ \\
\hline 11 & 5 Aug & 40.0 & bip & bip & $08^{\circ} 56^{\prime} 4 \mathrm{~N}, 147^{\circ} 58^{\prime} 7 \mathrm{E}$ \\
\hline 12 & 7 Aug & 27.6 & bip & bip & $01^{\circ} 41^{\prime} 6 \mathrm{~N}, 160^{\circ} 27^{\prime} 5 \mathrm{E}$ \\
\hline 13 & 7 Aug & 24.0 & bip & bip & $01^{\circ} 41^{\prime} 6 \mathrm{~N}, 160^{\circ} 27^{\prime} 5 \mathrm{E}$ \\
\hline 14 & 7 Aug & 27.8 & bip & bip & $01^{\circ} 41^{\prime} 6 \mathrm{~N}, 160^{\circ} 27^{\prime} 5 \mathrm{E}$ \\
\hline 15 & 7 Aug & 47.9 & bip & bip & $01^{\circ} 25^{\prime} 9 \mathrm{~N}, 160^{\circ} 54^{\prime} 0 \mathrm{E}$ \\
\hline 16 & $8 \mathrm{Aug}$ & 48.0 & bip & bip & $01^{\circ} 09^{\prime} 4 \mathrm{~N}, 161^{\circ} 21^{\prime} 5 \mathrm{E}$ \\
\hline 17 & 8 Aug & 41.2 & bip & bip & $01^{\circ} 09^{\prime} 4 \mathrm{~N}, 161^{\circ} 21^{\prime} 5 \mathrm{E}$ \\
\hline 18 & 8 Aug & 32.7 & bip & bip & $01^{\circ} 09^{\prime} 4 \mathrm{~N}, 161^{\circ} 21^{\prime} 5 \mathrm{E}$ \\
\hline 19 & 8 Aug & 28.1 & bip & bip & $01^{\circ} 09^{\prime} 4 \mathrm{~N}, 161^{\circ} 21^{\prime} 5 \mathrm{E}$ \\
\hline 20 & 13 Aug & 35.8 & Anguilla sp. & mar & $09^{\circ} 56^{\prime} 8 \mathrm{~S}, 174^{\circ} 59^{\prime} 7 \mathrm{E}$ \\
\hline 21 & 13 Aug & 42.8 & Anguilla sp. & meg & $09^{\circ} 56^{\prime} 8 \mathrm{~S}, 174^{\circ} 59^{\prime} 8 \mathrm{E}$ \\
\hline 22 & 15 Aug & 39.2 & Anguilla sp. & $\operatorname{mar}$ & $13^{\circ} 00^{\prime} 1 \mathrm{~S}, 174^{\circ} 60^{\prime} \mathrm{OE}$ \\
\hline 23 & $3 \mathrm{Sep}$ & 21.1 & meg or rei & rei & $14^{\circ} 30^{\prime} 9 \mathrm{~S}, 160^{\circ} 03^{\prime} 4 \mathrm{E}$ \\
\hline 24 & 3 Sep & 26.5 & meg or die & aua & $15^{\circ} 30^{\prime} 0 \mathrm{~S}, 160^{\circ} 00^{\prime} 2 \mathrm{E}$ \\
\hline 25 & 3 Sep & 47.3 & mar & mar & $15^{\circ} 04^{\prime} 3 \mathrm{~S}, 160^{\circ} 04^{\prime} 4 \mathrm{E}$ \\
\hline 26 & $3 \mathrm{Sep}$ & 33.7 & meg or die & rei & $14^{\circ} 30^{\prime} 9 \mathrm{~S}, 160^{\circ} 03^{\prime} 4 \mathrm{E}$ \\
\hline 27 & 3 Sep & 20.5 & meg & aua & $15^{\circ} 04^{\prime} 3 \mathrm{~S}, 160^{\circ} 04^{\prime} 4 \mathrm{E}$ \\
\hline 28 & 3 Sep & 26.3 & rei & rei & $14^{\circ} 30^{\prime} 9 \mathrm{~S}, 160^{\circ} 03^{\prime} 4 \mathrm{E}$ \\
\hline 29 & $3 \mathrm{Sep}$ & 25.0 & rei & rei & $15^{\circ} 04^{\prime} 3 \mathrm{~S}, 160^{\circ} 04^{\prime} 4 \mathrm{E}$ \\
\hline 30 & 3 Sep & 26.0 & rei & rei & $14^{\circ} 30^{\prime} 9 \mathrm{~S}, 160^{\circ} 03^{\prime} 4 \mathrm{E}$ \\
\hline 31 & 3 Sep & 22.5 & meg or rei & aua & $14^{\circ} 30^{\prime} 9 \mathrm{~S}, 160^{\circ} 03^{\prime} 4 \mathrm{E}$ \\
\hline 32 & $3 \mathrm{Sep}$ & 26.4 & rei & rei & $15^{\circ} 04^{\prime} 3 \mathrm{~S}, 160^{\circ} 04^{\prime} 4 \mathrm{E}$ \\
\hline 33 & 3 Sep & 22.4 & rei & aua & $14^{\circ} 30^{\prime} 9 \mathrm{~S}, 160^{\circ} 03^{\prime} 4 \mathrm{E}$ \\
\hline 34 & $4 \mathrm{Sep}$ & 22.0 & rei & rei & $14^{\circ} 30^{\prime} 9 \mathrm{~S}, 160^{\circ} 03^{\prime} 4 \mathrm{E}$ \\
\hline 35 & 4 Sep & 22.8 & rei & aua & $14^{\circ} 30^{\prime} 9 \mathrm{~S}, 160^{\circ} 03^{\prime} 4 \mathrm{E}$ \\
\hline 36 & 4 Sep & 34.0 & rei? & rei & $14^{\circ} 30^{\prime} 9 \mathrm{~S}, 160^{\circ} 03^{\prime} 4 \mathrm{E}$ \\
\hline 37 & 4 Sep & 22.4 & meg or rei & rei & $14^{\circ} 30^{\prime} 9 \mathrm{~S}, 160^{\circ} 03^{\prime} 4 \mathrm{E}$ \\
\hline 38 & 4 Sep & 28.6 & rei & rei & $14^{\circ} 30^{\prime} 9 \mathrm{~S}, 160^{\circ} 03^{\prime} 4 \mathrm{E}$ \\
\hline 39 & $4 \mathrm{Sep}$ & 32.2 & aua & aua & $14^{\circ} 30^{\prime} 9 \mathrm{~S}, 160^{\circ} 03^{\prime} 4 \mathrm{E}$ \\
\hline 40 & 4 Sep & 22.0 & meg or die & rei & $14^{\circ} 30^{\prime} 9 \mathrm{~S}, 160^{\circ} 03^{\prime} 4 \mathrm{E}$ \\
\hline 41 & $4 \mathrm{Sep}$ & 20.0 & meg or die & rei & $14^{\circ} 30^{\prime} 9 \mathrm{~S}, 160^{\circ} 03^{\prime} 4 \mathrm{E}$ \\
\hline 42 & $4 \mathrm{Sep}$ & 28.4 & meg or rei & aua & $14^{\circ} 30^{\prime} 9 \mathrm{~S}, 160^{\circ} 03^{\prime} 4 \mathrm{E}$ \\
\hline 43 & 4 Sep & 30.0 & aua & aua & $14^{\circ} 30^{\prime} 9 \mathrm{~S}, 160^{\circ} 03^{\prime} 4 \mathrm{E}$ \\
\hline 44 & 4 Sep & 32.2 & meg? & aua & $14^{\circ} 30^{\prime} 9 \mathrm{~S}, 160^{\circ} 03^{\prime} 4 \mathrm{E}$ \\
\hline 45 & 5 Sep & 41.7 & mar or obs & meg & $10^{\circ} 03^{\prime} 4 \mathrm{~S}, 161^{\circ} 02^{\prime} \mathrm{OE}$ \\
\hline 46 & 6 Sep & 36.3 & mar & $\operatorname{mar}$ & $05^{\circ} 57^{\prime} 5 \mathrm{~S}, 160^{\circ} 01^{\prime} 1 \mathrm{E}$ \\
\hline 47 & $7 \mathrm{Sep}$ & 48.3 & obs & bip & $02^{\circ} 02^{\prime} 7 \mathrm{~S}, 157^{\circ} 35^{\prime} 9 \mathrm{E}$ \\
\hline 48 & $7 \mathrm{Sep}$ & 25.2 & mar or obs & $\operatorname{mar}$ & $03^{\circ} 52^{\prime} 7 \mathrm{~S}, 158^{\circ} 59^{\prime} 5 \mathrm{E}$ \\
\hline 49 & $7 \mathrm{Sep}$ & 48.3 & obs & bip & $02^{\circ} 02^{\prime} 7 \mathrm{~S}, 157^{\circ} 35^{\prime} 9 \mathrm{E}$ \\
\hline 50 & $7 \mathrm{Sep}$ & 26.3 & mar or obs & $\operatorname{mar}$ & $03^{\circ} 52^{\prime} 7 \mathrm{~S}, 158^{\circ} 59^{\prime} 5 \mathrm{E}$ \\
\hline 51 & 7 Sep & 49.0 & obs & bip & $04^{\circ} 59^{\prime} 6 \mathrm{~S}, 160^{\circ} 00^{\prime} O \mathrm{E}$ \\
\hline 52 & 7 Sep & 21.3 & mar & $\operatorname{mar}$ & $02^{\circ} 02^{\prime} 7 \mathrm{~S}, 157^{\circ} 35^{\prime} 9 \mathrm{E}$ \\
\hline 53 & 7 Sep & 36.2 & mar & $\operatorname{mar}$ & $03^{\circ} 52^{\prime} 7 \mathrm{~S}, 158^{\circ} 59^{\prime} 5 \mathrm{E}$ \\
\hline 54 & 8 Sep & 54.1 & Anguilla $\mathrm{sp}$ & bip & $01^{\circ} 01^{\prime} 1 \mathrm{~S}, 156^{\circ} 49^{\prime} \mathrm{OE}$ \\
\hline 55 & $8 \mathrm{Sep}$ & 32.6 & bip & bip & $01^{\circ} 51^{\prime} 4 \mathrm{~N}, 154^{\circ} 32^{\prime} 0 \mathrm{E}$ \\
\hline 56 & $8 \mathrm{Sep}$ & 50.9 & Anguilla sp. & bip & $01^{\circ} 01^{\prime} 1 \mathrm{~S}, 156^{\circ} 49^{\prime} 0 \mathrm{E}$ \\
\hline 57 & 8 Sep & 49.9 & Anguilla sp. & bip & $01^{\circ} 01^{\prime} 1 \mathrm{~S}, 156^{\circ} 49^{\prime} 0 \mathrm{E}$ \\
\hline 58 & 8 Sep & 29.3 & bip & bip & $01^{\circ} 51^{\prime} 4 \mathrm{~N}, 154^{\circ} 32^{\prime} 0 \mathrm{E}$ \\
\hline 59 & $8 \mathrm{Sep}$ & 50.9 & Anguilla sp. & bip & $01^{\circ} 01^{\prime} 1 \mathrm{~S}, 156^{\circ} 49^{\prime} 0 \mathrm{E}$ \\
\hline 60 & $8 \mathrm{Sep}$ & 51.0 & Anguilla sp. & bip & $01^{\circ} 01^{\prime} 1 \mathrm{~S}, 156^{\circ} 49^{\prime} 0 \mathrm{E}$ \\
\hline 61 & 9 Sep & 34.1 & bip & bip & $02^{\circ} 19^{\prime} 6 \mathrm{~N}, 154^{\circ} 09^{\prime} 9 \mathrm{E}$ \\
\hline 62 & 10 Sep & 41.9 & obs & bip & $08^{\circ} 18^{\prime} 7 \mathrm{~N}, 149^{\circ} 42^{\prime} 9 \mathrm{E}$ \\
\hline 63 & $10 \mathrm{Sep}$ & 41.3 & obs & bip & $06^{\circ} 32^{\prime} 0 \mathrm{~N}, 151^{\circ} 01^{\prime} 1 \mathrm{E}$ \\
\hline
\end{tabular}




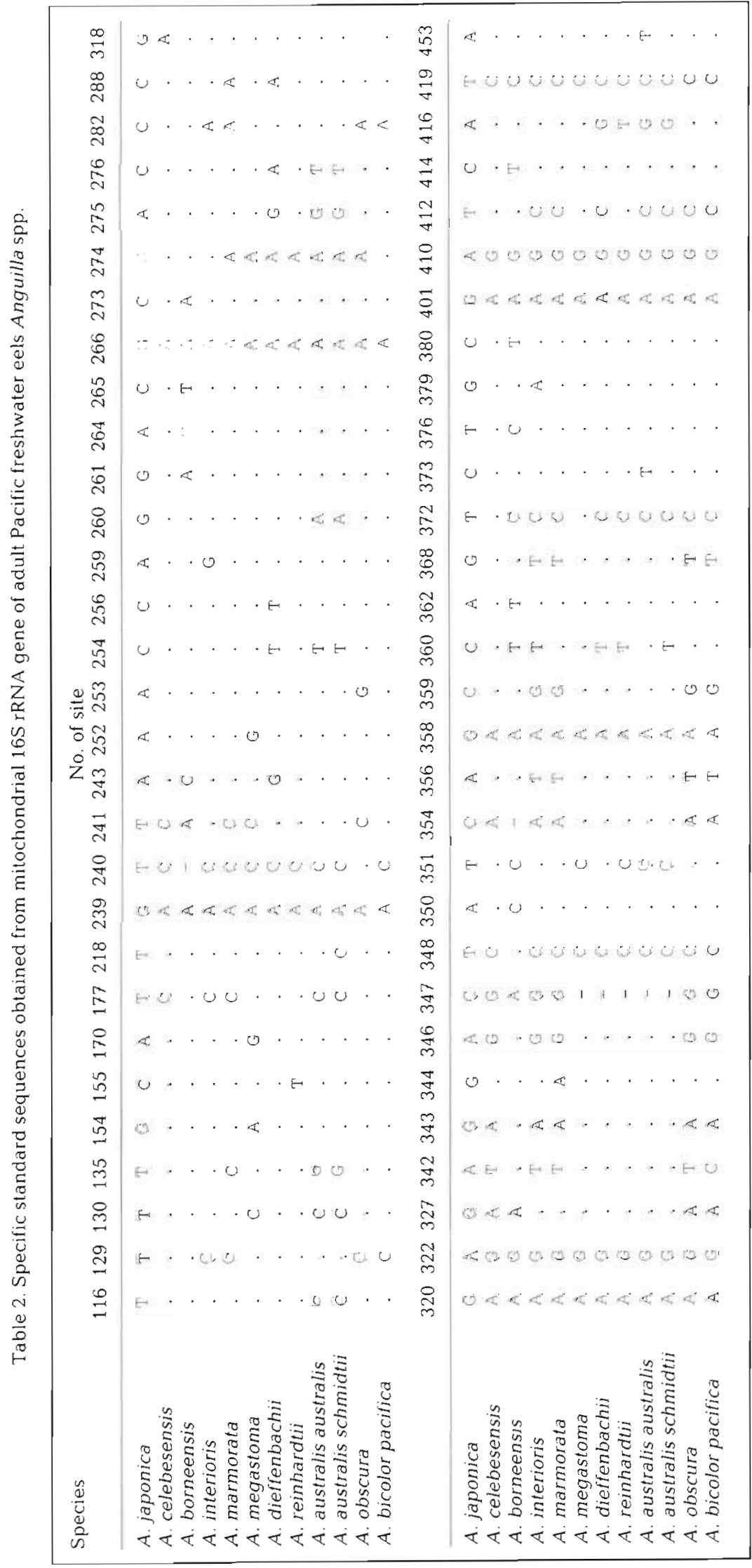

\section{DISCUSSION}

\section{Species identification}

Aoyama (1998) revealed that intra-species variation (less than $0.4 \%$ ) in the mitochondrial $16 \mathrm{~S}$ ribosomal RNA gene sequence was much less than inter-species variation for 2 species of freshwater eels, Anguilla marmorata and A. celebesensis, and suggested that specific standard sequences in this gene could be established from only 1 specimen of each species. Indeed, a sequence obtained from a leptocephalus agreed closely with 1 of the specific standard sequences with only slight variation (less than 3 sites).

The present study could not clearly distinguish the subspecies Anguilla australis australis and $A$. australis schmidtii, as recently reported by Dijkstra \& Jellyman (1999). Morphological differences between these subspecies were very slight and added further doubt to the validity of recognizing the 2 subspecies (Castle 1963, Tesch 1977, Jellyman 1987, Aoyama 1998). Furthermore, recent genetic investigation of all species of anguillid eels revealed that genetic differences between the adults of both subspecies could be entirely included in intra-species variation (Aoyama 1998). In consideration of the above, it can be concluded that the genetic difference observed between $A$, australis australis and $A$. australis schmidtii is attributed to intra-species variation of $A$ australis.

The concordance between molecular and morphological identification was $79.5 \%$. Critical misidentifications by morphological characters used in the present study were mostly for leptocephali smaller than $30 \mathrm{~mm}$ total length (TL) (Table 1). The number of myomeres is the most important for the species identification of leptocephali (Jespersen 1942). However, it is well 
known that the number of myomeres varies somewhat within species, and this number is difficult to determine in small leptocephali with morphological characteristics being not fully developed (Mochioka 1996). A molecular key is applicable to any developmental stages from eggs to adults. Species identification of small anguillid leptocephali cannot be made without consideration of molecular data, particularly in areas where several species occur sympatrically, as in the South Pacific.

\section{Distribution and migration of anguillid leptocephali}

The horizontal distributions of the anguillid leptocephali studied here are closely related to the freshwater habitat of the adults by way of ocean currents (Fig. 2), as previously inferred by Tsukamoto \& Aoyama (1998). For example, Anguilla marmorata leptocephali have a patchy distribution in the North Equatorial Current, and probably disperse to eastern Asia by the North Equatorial Current and further by the Kuroshio Current. The A. marmorata leptocephali occurring patchily in the South Equatorial Current would be transported to northern New Guinea, New Caledonia and Fiji by the South Equatorial Current. This patchiness corresponds well to North Pacific and western South Pacific populations, respectively, as revealed by the molecular analysis of $A$. marmorata throughout the Indo-Pacific (Ishikawa 1998).

Anguilla bicolor pacifica leptocephali found near the boundary between the Equatorial Counter Current and the South Equatorial Current showed a different distribution to those of A. marmorata, although their adults occur sympatrically. This suggests the existence of different currents which transport leptocephali from 2 different areas to the same coastal region.
Table 3. Number of differences between standard specific sequences and homologous sites of anguillid leptocephali. Species names are abbreviated as follows: jap: $A$. japonica, cel: A. celebesensis, rei: A. reinhardti, obs. A. obscura, meg: A. megastoma, mar: A. marmorata, int: $A$. interioris, die: $A$. dieffenbachii, bor: $A$. borneensis, bip: $A$. bicolor pacifica, aus: A. australis schmidtii, aua: A. australis australis

\begin{tabular}{|c|c|c|c|c|c|c|c|c|c|c|c|c|}
\hline Fish no. & jap & cel & rei & obs & meg & $\operatorname{mar}$ & int & die & bor & bip & aus & aua \\
\hline 01 & 25 & 12 & 16 & 7 & 17 & 0 & 8 & 17 & 27 & 8 & 20 & 18 \\
\hline 02 & 25 & 12 & 16 & 7 & 17 & 0 & 8 & 17 & 27 & 8 & 20 & 18 \\
\hline 03 & 25 & 12 & 16 & 7 & 17 & 0 & 8 & 17 & 27 & 8 & 20 & 18 \\
\hline 04 & 26 & 13 & 17 & 8 & 18 & 1 & 9 & 18 & 28 & 9 & 19 & 17 \\
\hline 05 & 0 & 20 & 18 & 23 & 19 & 26 & 24 & 24 & 25 & 20 & 27 & 25 \\
\hline 06 & 25 & 12 & 16 & 7 & 17 & 0 & 8 & 17 & 27 & 8 & 20 & 18 \\
\hline 07 & 25 & 12 & 16 & 7 & 18 & 0 & 8 & 17 & 27 & 8 & 20 & 18 \\
\hline 08 & 20 & 10 & 13 & 5 & 16 & 8 & 6 & 16 & 21 & 0 & 20 & 18 \\
\hline 09 & 20 & 10 & 13 & 5 & 16 & 8 & 6 & 16 & 21 & 0 & 20 & 18 \\
\hline 10 & 20 & 10 & 13 & 5 & 17 & 8 & 6 & 16 & 21 & 0 & 20 & 18 \\
\hline 11 & 21 & 11 & 14 & 6 & 18 & 9 & 7 & 17 & 22 & 1 & 21 & 19 \\
\hline 12 & 20 & 10 & 13 & 5 & 16 & 8 & 6 & 16 & 21 & 0 & 20 & 18 \\
\hline 13 & 20 & 10 & 13 & 6 & 17 & 9 & 7 & 16 & 21 & 1 & 20 & 18 \\
\hline 14 & 20 & 10 & 13 & 5 & 17 & 8 & 6 & 16 & 21 & 0 & 20 & 18 \\
\hline 15 & 20 & 10 & 13 & 5 & 17 & 8 & 6 & 16 & 21 & 0 & 20 & 18 \\
\hline 16 & 20 & 10 & 13 & 5 & 17 & 8 & 6 & 16 & 21 & 0 & 20 & 18 \\
\hline 17 & 20 & 10 & 13 & 5 & 17 & 8 & 6 & 16 & 21 & 0 & 20 & 18 \\
\hline 18 & 21 & 11 & 14 & 6 & 18 & 9 & 7 & 17 & 22 & 1 & 21 & 19 \\
\hline 19 & 20 & 10 & 13 & 5 & 16 & 8 & 6 & 16 & 21 & 0 & 20 & 18 \\
\hline 20 & 25 & 12 & 16 & 7 & 17 & 0 & 8 & 17 & 27 & 8 & 20 & 18 \\
\hline 21 & 24 & 16 & 14 & 21 & 3 & 22 & 24 & 21 & 25 & 21 & 21 & 19 \\
\hline 22 & 25 & 12 & 16 & 7 & 17 & 0 & 8 & 17 & 27 & 8 & 20 & 18 \\
\hline 23 & 20 & 11 & 2 & 15 & 10 & 18 & 16 & 12 & 16 & 13 & 14 & 13 \\
\hline 24 & 25 & 17 & 12 & 20 & 14 & 18 & 19 & 11 & 25 & 18 & 2 & 0 \\
\hline 25 & 25 & 12 & 16 & 7 & 18 & 0 & 8 & 17 & 27 & 8 & 20 & 18 \\
\hline 26 & 18 & 12 & 0 & 15 & 10 & 16 & 16 & 10 & 16 & 13 & 12 & 12 \\
\hline 27 & 26 & 18 & 11 & 21 & 16 & 19 & 20 & 10 & 24 & 19 & 1 & 1 \\
\hline 28 & 18 & 12 & 0 & 15 & 9 & 16 & 16 & 10 & 16 & 13 & 12 & 12 \\
\hline 29 & 19 & 12 & 1 & 16 & 9 & 17 & 15 & 11 & 17 & 14 & 13 & 12 \\
\hline 30 & 18 & 12 & 0 & 15 & 10 & 16 & 16 & 10 & 16 & 13 & 12 & 12 \\
\hline 31 & 26 & 17 & 11 & 21 & 14 & 19 & 18 & 12 & 24 & 19 & 3 & 2 \\
\hline 32 & 21 & 14 & 3 & 18 & 12 & 19 & 17 & 13 & 19 & 16 & 15 & 14 \\
\hline 33 & 26 & 18 & 11 & 21 & 15 & 19 & 20 & 10 & 24 & 19 & 1 & 1 \\
\hline 34 & 20 & 14 & 2 & 17 & 12 & 18 & 18 & 12 & 18 & 15 & 14 & 14 \\
\hline 35 & 27 & 21 & 14 & 22 & 19 & 22 & 23 & 13 & 26 & 22 & 3 & 3 \\
\hline 36 & 21 & 15 & 3 & 17 & 12 & 18 & 18 & 13 & 19 & 15 & 15 & 15 \\
\hline 37 & 19 & 12 & 1 & 16 & 9 & 17 & 15 & 11 & 17 & 14 & 13 & 12 \\
\hline 38 & 19 & 12 & 1 & 16 & 9 & 17 & 15 & 11 & 17 & 14 & 13 & 12 \\
\hline 39 & 26 & 18 & 11 & 21 & 16 & 19 & 20 & 10 & 24 & 19 & 1 & 1 \\
\hline 40 & 18 & 12 & 0 & 15 & 10 & 16 & 16 & 10 & 16 & 13 & 12 & 12 \\
\hline 41 & 18 & 12 & 0 & 15 & 10 & 16 & 16 & 10 & 16 & 13 & 12 & 12 \\
\hline 42 & 27 & 18 & 12 & 22 & 15 & 20 & 19 & 11 & 25 & 20 & 2 & 1 \\
\hline 43 & 27 & 18 & 12 & 22 & 15 & 20 & 19 & 11 & 25 & 20 & 2 & 1 \\
\hline 44 & 26 & 18 & 11 & 21 & 15 & 19 & 20 & 10 & 24 & 19 & 1 & 1 \\
\hline 45 & 21 & 12 & 9 & 18 & 0 & 19 & 21 & 16 & 20 & 18 & 16 & 15 \\
\hline 46 & 25 & 12 & 16 & 7 & 18 & 0 & 8 & 17 & 27 & 8 & 20 & 18 \\
\hline 47 & 20 & 10 & 13 & 5 & 17 & 8 & 6 & 16 & 21 & 0 & 20 & 18 \\
\hline 48 & 25 & 12 & 16 & 7 & 17 & 0 & 8 & 17 & 27 & 8 & 20 & 18 \\
\hline 49 & 20 & 10 & 13 & 5 & 16 & 8 & 6 & 16 & 21 & 0 & 20 & 18 \\
\hline 50 & 25 & 12 & 16 & 7 & 17 & 0 & 8 & 17 & 27 & 8 & 20 & 18 \\
\hline 51 & 20 & 10 & 13 & 5 & 16 & 8 & 6 & 16 & 21 & 0 & 20 & 18 \\
\hline 52 & 25 & 12 & 16 & 7 & 18 & 0 & 8 & 17 & 27 & 8 & 20 & 18 \\
\hline 53 & 25 & 12 & 16 & 7 & 17 & 0 & 8 & 17 & 27 & 8 & 20 & 18 \\
\hline 54 & 20 & 10 & 13 & 6 & 16 & 9 & 7 & 16 & 21 & 1 & 20 & 18 \\
\hline 55 & 20 & 10 & 13 & 5 & 17 & 8 & 6 & 16 & 21 & 0 & 20 & 18 \\
\hline 56 & 21 & 11 & 14 & 6 & 18 & 9 & 7 & 17 & 22 & 1 & 21 & 19 \\
\hline 57 & 21 & 11 & 14 & 6 & 17 & 9 & 7 & 17 & 22 & 1 & 21 & 19 \\
\hline 58 & 20 & 10 & 13 & 5 & 16 & 8 & 6 & 16 & 21 & 0 & 20 & 18 \\
\hline 59 & 21 & 11 & 14 & 6 & 18 & 9 & 7 & 17 & 22 & 1 & 21 & 19 \\
\hline 60 & 20 & 10 & 13 & 5 & 16 & 8 & 6 & 16 & 21 & 0 & 20 & 18 \\
\hline 61 & 20 & 10 & 13 & 5 & 16 & 8 & 6 & 16 & 21 & 0 & 20 & 18 \\
\hline 62 & 20 & 10 & 13 & 5 & 17 & 8 & 6 & 16 & 21 & 0 & 20 & 18 \\
\hline 63 & 20 & 10 & 13 & 5 & 16 & 8 & 6 & 16 & 21 & 0 & 20 & 18 \\
\hline
\end{tabular}




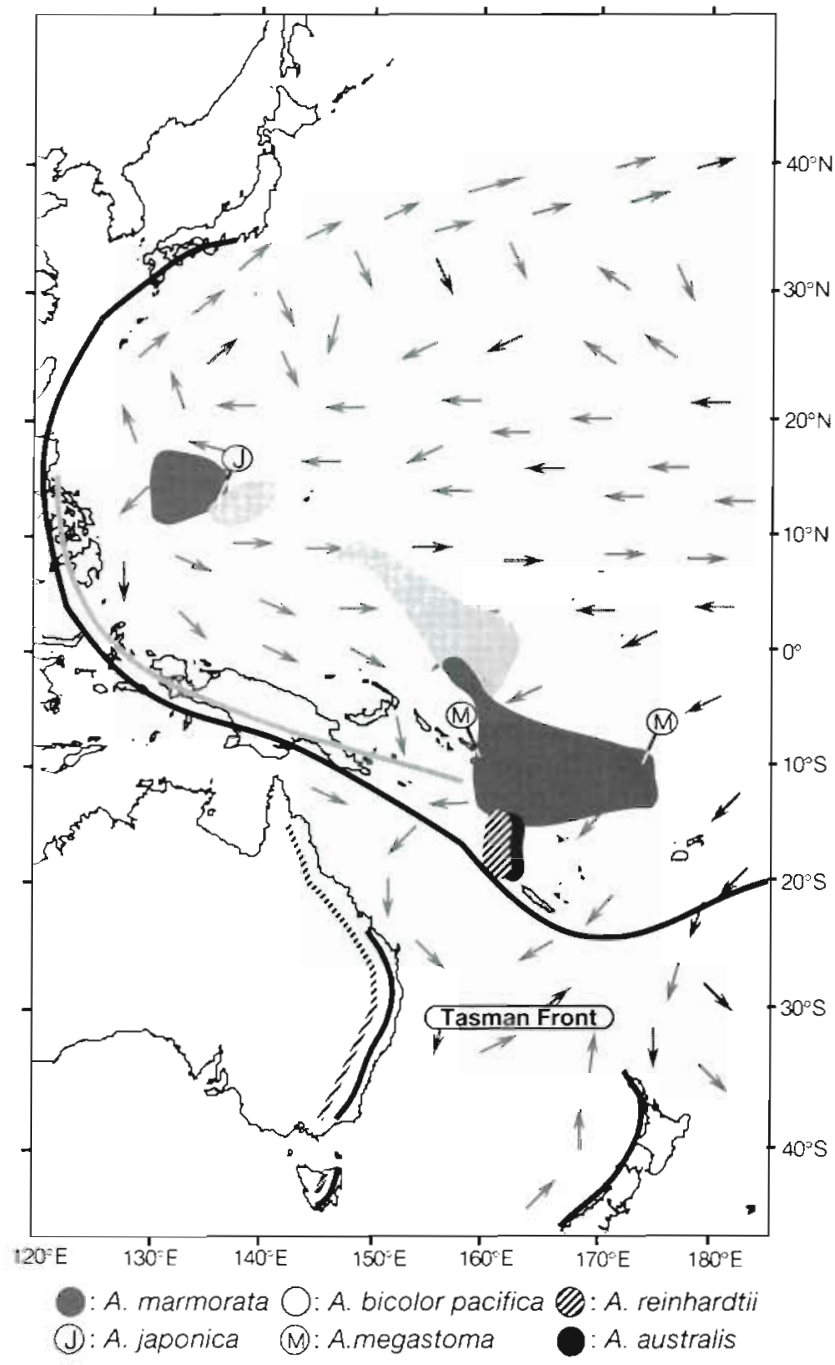

Fig. 2. Distribution of anguillid leptocephali in the western Pacific and their adult freshwater distributions (correspondingly shaded bars)

The distributions of leptocephali of Anguilla reinhardtii and A. australis completely overlap and the difference in their TLs was not significant (Fig. 3). These findings suggest that they could be transported to the same area by overlapping dispersal routes, although their adult distributions do not completely coincide. The northern part of the east coast of Australia is populated by only $A$. reinhardtii, and in the southern part both species live sympatrically (Jellyman 1987, Sloane 1984). A previous study in New South Wales (Ege 1939), where both species are found, suggested that the TLs of recruiting glass eels of $A$. reinhardtii ( 46 to $65 \mathrm{~mm}$ ) were slightly less than A. australis (47 to $73 \mathrm{~mm}$ ). These data indicate that $A$. reinhardtii has a shorter larval duration and detrains from the East Australian Current earlier than A. australis, resulting in the solitary recruitment of $A$. rein- hardtii to the northern part of Australia. Some of $A$. reinhardtii which have relatively long larval duration within intraspecies variation might be transported to the southern part of Australia and further to New Zealand, as has been recently revealed (Jellyman et al. 1996, McDowall et al. 1998).

These observations strongly suggest that leptocephali of freshwater eels in the western Pacific have specific dispersal routes from their adult spawning areas to their freshwaters. Even if a part of their larval dispersal routes overlap, there is likely to be a mechanism to transport leptocephali/glass eels to the specific distributional ranges of their adults. Specific larval durations might be the key to the nature of such movements, as previously suggested for the Atlantic eels (Schmidt 1923, 1925).

In New Zealand, where Anguilla dieffenbachii and A. australis are in general sympatric, glass eels of both species migrate to estuaries from August to November (Jellyman 1987). Assuming that they spawn in different locations but that they utilize partly overlapped dispersal routes, the leptocephali might appear sympatrically. However, A. dieffenbachii leptocephali were not collected in the present study. Jellyman (1987) suggested that $A$. dieffenbachii might have its spawning area nearer to land than $A$. australis since the maturation index of female silver eels migrating to the sea is higher in the former (8.12) than in the latter (3.49, Jellyman 1987). Although the lack of leptocephali of $A$. dieffenbachii in the present collections
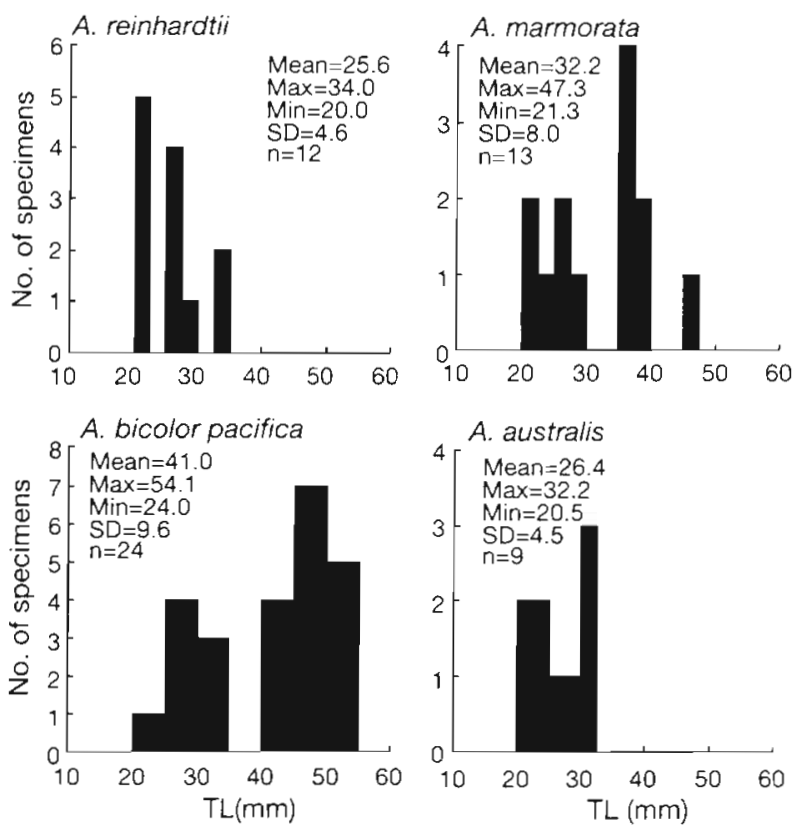

Fig. 3. Distribution of total length of anguillid leptocephalus examined in the present study. One Anguilla japonica $28.1 \mathrm{~mm}$ and 2 A. megastoma $42.8,41.7 \mathrm{~mm}$ were removed from the figure 
may not mean that they are absent from the area, it seems reasonable to suppose that the migration route for $A$. dieffenbachii lies out of the area searched here. The absence of leptocephali of $A$. celebesensis, $A$. borneensis and $A$. obscura in the present study might be due to a mismatch of their migration route and/or spawning time.

\section{Spawning area of Anguilla australis}

Based on the collection of 4 leptocephali, with additional information on oceanic hydrography, size of glass eels and their arrival times in freshwater, several hypotheses concerning the possible spawning area of Anguilla australis have been proposed. Castle (1963) and Tesch (1977) speculated that the area might be between Fiji and Tahiti, while Schmidt (1928) placed it on the western side of New Caledonia. Jellyman (1987) suggested that the spawning area for $A$. australis lies across the area of divergence of the South Equatorial Current, 150 to $170^{\circ} \mathrm{W}$ and 5 to $15^{\circ} \mathrm{S}$. Larvae in the northern part of the spawning area would then tend to be transported to the western Tasman Sea, and hence to the Australian coast, while those in the southern part might be dispersed to New Zealand. In the present study, however, no anguillid leptocephali were collected south of New Caledonia, while all of the A. australis were caught north of New Caledonia, suggesting that the $A$. australis recruiting to New Zealand follow a different route. It is likely that a part of $A$. australis dispersing to the northeast coast of Australia via north of New Caledonia could recruit further to New Zealand, using the eastward zonal flow of the Tasman Front latitudinally across the Tasman Sea at about $30^{\circ} \mathrm{S}$. It might reflect the difference of arrival time of glass eels, February to November in Australia and August to November in New Zealand (Jellyman 1987, Sloane 1984).

If it is assumed that the growth rate of Anguilla australis is the same as those calculated for $A$. japonica, i.e. $0.43 \mathrm{~mm} \mathrm{~d}^{-1}$ (Tsukamoto et al. 1994), the leptocephali of $A$, australis collected in the present study (at about $14^{\circ} \mathrm{S}$ and $160^{\circ} \mathrm{E}$, average $26.4 \mathrm{~mm} \mathrm{TL}$ ) are therefore likely to be ca $54 \mathrm{~d}$ old. This suggests that these leptocephali could be transported ca $1866 \mathrm{~km}$ westward from a possible eastern spawning area, by the South Equatorial Current $\left(40 \mathrm{~cm} \mathrm{~s}^{-1}\right.$, Jellyman 1987). Furthermore time needs to be allowed for the transport of developing eggs

In consideration of the above, the location of the spawning area for Anguilla australis might be inferred to agree with Castle's speculation (Castle 1963). Further investigations in this area will be required to determine more confidently the precise spawning area of $A$. australis.
Acknowledgements. We thank Dr M. Miller for cooperation in the morphological identification of leptocephali. The authors are grateful to the crew of RV 'Hakuho-Maru', Ocean Research Institute, University of Tokyo. J.A. was partly supported by Research Fellowships of the Japan Society for the Promotion of Science for Young Scientists. This study was supported partly by Grant-in-aid (Nos. 08456094, 10460081, and 08041139) from the Ministry of Education, Science and Culture of Japan; 'Research for the Future' Program No. JSPS-RFTF 97L00901 from the Japan Society for the Promotion of Science; Touwa Shokuhin Shinkoukai and by Eel Research Foundation from Nobori-kai.

\section{LITERATURE CITED}

Aoyama J (1998) Molecular phylogeny and evolution of the freshwater eels, genus Anguilla. PhD thesis, University of Tokyo

Aoyama J, Tsukamoto K (1997) Evolution of the freshwater eels. Naturwissenschaften 84:17-21

Castle PHJ (1963) Anguillid leptocephali in the southwest Pacific. Zoology Publications, Victoria University of Wellington 33:1-14

Dijkstra LH, Jellyman DJ (1999) Is the subspecies classification of the freshwater eels Anguilla australis australis Richardson and A. a. schmidtii Phillipps still valid? Mar Freshw Res 50:261-263

Ege V (1939) A revision of the genus Anguilla shaw, a systematic, phylogenetic and geographical study. Dana-Rep 16:1-256

Ishikawa S (1998) Molecular study on the population structure of Anguilla marmorata. PhD thesis, University of Tokyo

Jellyman DJ (1987) Review of the marine life history of Australian temperate species of Anguilla. In: Dadwell MJ, Klauda RJ, Moffitt CM, Saunders RL, Rulifson RA, Cooper JE (eds) Common strategies of anadromous and catadromous fishes. American Fisheries Society, MA, p 276-285

Jellyman DJ, Dijkstra LH, Chisnall BW, Boubee JAT (1996) The first record of the Australasian longfinned eel Anguilla reinhardtii, in New Zealand. Mar Freshw Res 47 : $1037-1040$

Jespersen P (1942) Indo-pacific leptocephalids of the genus Anguilla. Dana-Rep 22:1-128

Kleckner RC, McCleave JD (1985) Spatial and temporal distribution of American eel larvae in relation to north Atlantic Ocean current systems. Dana 4:67-92

Maddison WP, Maddison DR (1992) MacClade Version 3. Sinauer Associates Inc, MA

McDowall RM, Jellyman DJ, Dijkstra LH (1998) Arrival of an Australian anguillid eel in New Zealand: an example of transoceanic dispersal. Environ Biol Fishes 51:1-6

Miya M, Nishida M (1996) Molecular phylogenetic perspective on the evolution of the deep-sea fish genus Cyclothone (Stomiiformes: Gonostomatidae). Ichthyol Res 43:375-398

Mochioka N (1996) Morphology and growth of Japanese eel larvae. In: Tabeta $\mathrm{O}(\mathrm{ed})$ Early life-history and prospects of seed production of the Japanese eel Anguilla japonica. Koseisya-koseikaku, Tokyo, p 22-32

Schmidt J (1923) The breeding places of the eel. Phil Trans Royal Soc 211:179-208

Schmidt J (1925) The breeding places of the eel. Annu Rep Smithsonian Inst 1924:279-316

Schmidt J (1928) The freshwater eels of Australia with some 
remarks on the shortfinned species of Anguilla. Rec Aust Mus 16:179-210

Sloane RD (1984) Invasion and upstream migration by glasseels of Anquilla australis australis Richardson and A. reinhardtii Steindachner in Tasmanian freshwater streams. Aust J Mar Freshw Res 35:47-59

Smith DG (1989) Order Angulliformes, family Anguillidae. In: Bohlke EB (ed) Fishes of the western North Atlantic. Sears Foundation for Marine Research, New Haven, CT, p $25-47$

Swofford DL (1991) PAUP: Phylogenetic analysis using parsimony, Version 3.0. Illinois natural history survey, Champaign, IL
Tabeta O, Mochioka N (1988) Leptocephali. In: Okiyama M (ed) An atlas of the early stage fishes in Japan. Tokai Univ Press, Tokyo, p 15-64

Tesch FW (1977) The eel. Chapman and Hall, London

Tsukamoto K (1992) Discovery of the spawning area for Japanese eel. Nature 356(6372):789-791

Tsukamoto K, Lee TW, Mochioka N (1994) Age and growth of Japanese eel leptocephali. In: Otake T, Tsukamoto K (eds) Preliminary report of the Hakuho Maru cruise KH-91-4. Ocean Research Institute, University of Tokyo, p 50-54

Tsukamoto K, Aoyama J (1998) Evolution of the freshwater eels of the genus Anguilla: probable scenario. Environ Biol Fishes 52(3): 139-148

Submitted: December 29, 1998; Accepted: May 7, 1999 Proofs received from author(s): October 8, 1999
Editorial responsibility: Otto Kinne (Editor),

Oldendorf/Luhe, Germany 